Research Paper:

\title{
Sleep Quality of Patients Admitted to the Coronary Care crosshark Unit: Pilot Study
}

Tahereh Najafi Ghezeljeh ${ }^{1}$, Maryam Nasari ${ }^{1 *}$

1. Department of Critical Care Nursing, School of Nursing and Midwifery, Iran University of Medical Sciences, Tehran, Iran.

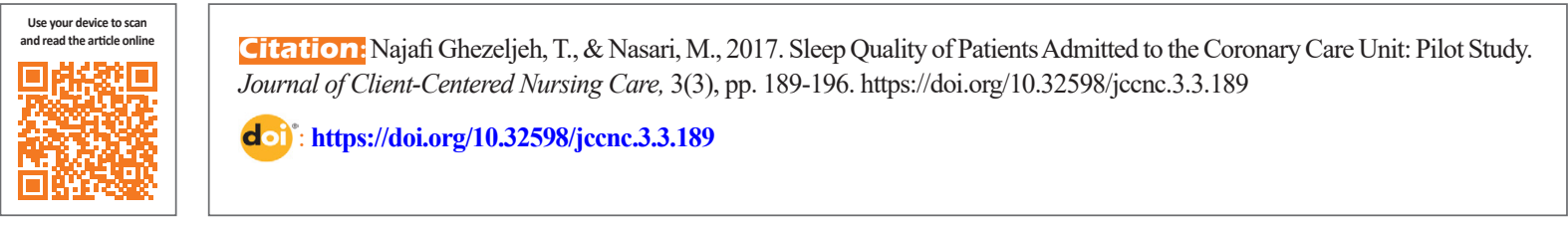

Article info:

Received: 14 Dec. 2016

Accepted: 19 Mar. 2017

Keywords:

Sleep quality, Hospital, Sleep deprivation,

Coronary care unit

\begin{abstract}
A B S T R A C T
Background: Sleep deprivation has numerous negative consequences on the human body and mind. It exacerbates anxiety, irritability, and nervousness and worsens the cardiovascular function. This study aimed at evaluating the quality of sleep in patients admitted to the cardiac intensive care unit.

Methods: This was a descriptive study with 93 patients hospitalized in critical care units of three teaching hospitals in 2016. The samples were chosen using a convenient sampling method. The quality of sleep was assessed in two consecutive days using the demographic data form and the Richards-Campbell Sleep Questionnaire (RCSQ). Descriptive and inferential statistics was used for data analysis via the SPSS software version 20.

Results: It was found that more than $90 \%$ of the patients in the cardiac care units had a low level of quality of sleep. The patients had problems in the depth of sleep, ease in falling asleep, frequent waking from sleep, ease in returning to sleep, and subjective sleep quality. The mean scores of the quality of sleep before hospitalization and in the first night after hospitalization were $63.68(\mathrm{SD}=17.96)$ and $54.2(\mathrm{SD}=16.90)$, respectively. A statistically significant correlation was reported between the quality of sleep in the night before and the first night of hospitalization ( $\mathrm{P}$ $<0.0001)$.
\end{abstract}

Conclusion: Due to the high prevalence of sleep disorders in patients admitted to cardiac intensive care units and the consequences of low sleep quality on their health, appropriate interventions for improving the quality of sleep in patients admitted to coronary care units are required.

\section{Background}

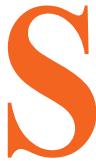

leep is a vital function in maintaining mental health, restoring physical fitness, and improving the condition of the body (Duong Coburn 2013). Many biological func- tions of the body, hormonal secretion, and functioning of the immune system changes during sleep as compared to when awake. Any disorder in the quantity, quality, or pattern of sleep can have a significant negative impact on the performance as well as physical and psychological health. Sleep disorders lead to an increased risk of cardiovascular

* Corresponding Author:

Maryam Nasari, MSc. Student

Address: Department of Critical Care Nursing, School of Nursing and Midwifery, Iran University of Medical Sciences, Tehran, Iran.

Tel: +98 (917) 0107917

E-mail: maryamnasari65@yahoo.com 
disease and vice versa; such diseases lead to physiological changes in sleep (Gami et al. 2013; Kendzerska et al. 2014; Tobaldini et al. 2017). A cross-sectional study of 475,000 people in the age group of 7-25 years showed that sleep disorders increase the risk of death from coronary heart disease by $48 \%$ (Paddoc 2013). Sleep deprivation as a stressful condition increases the sympathetic tone, followed by epinephrine and norepinephrine secretion, which ultimately exacerbates ischemia and cardiac infarction (Kozick 2013). Also, in the psychological aspect, it can cause physical stress, agitation, aggression, reduced physical and cognitive performance, mood instability, and emotional disturbances in patients (Fontana \& Pittiglio 2010). In other words, sleep disorder causes physiological changes in the body and endangers the health of the patient (Schiza \& Mermigkis 2010).

Early diagnosis of sleep disorder is necessary to reduce the possible risks. Nurses must help patients to improve their sleep quality through subjective and objective examinations and early diagnosis of insomnia and also in designing interventions. The nature of the disease and its complications, pain, stress, anxiety, underlying diseases, drugs and individual factors, physical disabilities, environmental factors of the Intensive Care Unit such as light, disturbances in the dark-light cycle (Adib Hajbaghery, Izadi Avanji \& Akbari 2012; Stewart et al., 2017) and sound (in particular, frequent and unpleasant sounds of mechanical ventilation, telephone ringing, coughing of other patients, opening and closing of windows and doors, cleaning of room, and talking of staff) are among the factors that can play a role in sleep disorder of patients (Fontana \& Pittiglio 2010; Little et al., 2012).

Several studies have been conducted on sleep quality in the Intensive Care Unit. Some reported the patients' sleep quality as undesirable (Ahmed et al. 2015; Bahame mam et al. 2010; Kamdar et al. 2013; Li et al. 2011), and some others reported no change or better than pre-admission (Monteiro \& Ceolim 2014). Despite the need for good quality sleep by most patients, a study found that most patients in the Coronary Care Unit suffered from sleep disorder (Chandola et al., 2010). Considering the importance of sleep and the contradictory results of previous studies, the present research examined the sleep quality of patients admitted to the Coronary Care Unit.

\section{Materials and Methods}

\section{Study design and participants}

This is a descriptive study of patients admitted to the Coronary Care Unit of Rasool Akram, Firoozgar, and
Rajai Shahr Hospitals in 2016. The study population consisted of all patients admitted to the Coronary Care Unit. The available sampling method was used in the study. The inclusion criteria included being at least 18 years old, admission to the Coronary Care Unit, having no known history of sleep disorder and hearing impairment, non-use of anesthetic drugs and antidepressants, and awareness of time, place, and person. To determine the sample size at $95 \%$ confidence level, the test power of $80 \%$ of the sample size was estimated to be 93 .

\section{Data collection}

Data collection tools included demographic information form and Richards-Campbell Sleep Questionnaire (RCSQ). The demographic information form was completed by examining the patient's file or asking questions. The RCSQ was used to measure their sleep quality. This questionnaire was developed by Richards et al. (2000), and its content validity was estimated using Cronbach's alpha coefficient of $82 \%$. This questionnaire was first found with searching words such as sleep quality and sleep quality questionnaire. Then, the questionnaire was translated into Persian by a professional translator in order to confirm the formal and content validity. It should be noted that the translation was carried out conceptually, not lexically.

The Persian version of the questionnaire was provided to 10 nursing professors to confirm its content validity. The questionnaire was provided to 10 patients in the CCU to determine its reliability and internal consistency. The Cronbach's alpha coefficient was obtained to be 0.96 . This scale has five items: 1) Sleep depth, 2) Ease of falling asleep, 3) Waking up frequently, 4) Ease of returning to sleep, and 5) Mental sleep quality (sleep quality from the patient's point of view). Each item had a visual scale of 0 to $100 \mathrm{~mm}$, and the participant was required to mark the response to each item on the scale. The total sleep quality is calculated on average from 100 points. The zero score shows the worst sleep quality and 100 represents the best sleep quality.

\section{Intervention}

The researcher visited the research environments after obtaining permission from the Ethics Committee and the research authorization from the Iran University of Medical Sciences. The research objectives and the procedure were explained to the hospital authorities, and they agreed to volunteer. Then, the patients 
conforming to the inclusion criteria were selected by the available sampling method and were given a full explanation about the research objectives. Patients willing to participate in the research completed a written informed consent form. The researcher visited the patients for two days and asked the patients to complete the RCSQ to assess the quality of their last night's sleep. Assessment of the sleep quality of the first night was related to the sleep quality of the patient the night before admission, and the assessment of the second night was related to the sleep quality of the patient on the first night of hospitalization. Assistant researcher completed the questionnaire on behalf of illiterate patients by asking them the questions present in the form.

\section{Data analysis}

Data were analyzed using SPSS software version 20. For this purpose, descriptive statistics (frequency, the percentage of frequency, mean, and standard deviation) were used. Also, inferential statistics (independent ttest, one-way ANOVA, Kruskal-Wallis test, and Pearson correlation coefficient) were used to determine the significance. The significance level was less than 0.05 .

\section{Results}

The study results of 93 patients admitted to the $\mathrm{CCU}$ indicated that the mean age of the subjects was 55.83 \pm 15.86 years. Most of the subjects $(72.80 \%)$ were married, $40 \%$ of them had a fair economic status, and $75.30 \%$ of the subjects were non-smokers. The results showed that there was no significant relationship between sleep quality and demographic variables (Table 1). The mean sleep time in the hospital was $1.65 \pm 0.88$.

In this study, the highest frequency was related to acute coronary syndrome (32.3\%) and the lowest frequency to acute pulmonary edema (8.6\%). Also, 17.2 $\%$ were related to other diseases that were included in this group due to their low frequency. The results showed that there was no statistically significant relationship between sleep quality and type of disease (Table 2). Based on the results, the patients had problems in sleeping depth, ease of falling asleep, waking up frequently, ease of returning to sleep, and quality of mental sleep, respectively (Table 3).

Table 4 shows the patients' sleep quality in the hospital before and at home. The comparison of the quality of sleep in the night before admission and the first night of admission showed a significant difference. The sleep quality on the first night of admission was more unfavorable than at home. The results of Pearson correlation test showed that there was a significant direct correlation between sleep quality at night before admission and the first night of admission. The mean of sleep quality before admission was $63.68 \pm 17.96$ and that on the first night of admission was $54.02 \pm$ $16.9(\mathrm{P}<0.0001)$.

\section{Discussion}

The present study revealed that the mean sleep quality of patients in the special department was low and more than $90 \%$ of the units had poor sleep quality. The unfamiliar, noisy, and stressful environment of the Intensive Care Unit and the frequent care interventions have been associated with sleep disorders such as lack of deep sleep and discontinuous sleep with frequent waking up. The results of this study are consistent with the results of the Matthews' study, which states that a significant percentage of patients in the Intensive Care Unit had poor sleep quality and had a period of delayed falling asleep and frequent waking up (Matthews, 2011).

Another study suggests that sleep quality in patients with acute coronary syndrome decreases over the first few days (Bahammam et al., 2010). It was also reported in a study that the sleeping pattern of patients in the Intensive Care Unit is abnormal, associated with frequent interruptions, changes in circadian rhythm, sleep deprivation, and a reduction in the Rapid Eye Movement (REM) stage, and about $50 \%$ of their sleep occurs during the day. In addition, $19 \%$ of them suffer from sleep disorder during the first year after release from hospital (Kamdar et al., 2013). According to a study, $50 \%$ of the patients reported significant sleep disorders during their hospitalization period, and the factors such as age, psychological factors, and self-illness may exacerbate sleep disorders (Achury-Saldana \& Achury 2010). Also, the present study results on poor sleep quality of patients in the Intensive Care Unit (Table 4) were consistent with the results of Zeighami and Shahparian (2013). Their study examined sleep disorders in patients with systolic heart failure, in which all patients with heart failure had low sleep quality and $53 \%$ had mild daily sleepiness (Zeighami \& Shahparian, 2013).

Also, the results of another study showed that there is evidence of significant sleep disorder in patients admitted to the Intensive Care Unit with poor sleep quality and decreased REM sleep (Elliott et al., 2011). The results of the study conducted by Neyse et al. (2011) showed that $63.35 \%$ of patients had poor sleep quality. Also, the results of a study showed 
Table 1. Frequency distribution of demographic characteristics and numerical indicators of sleep quality according to these characteristics and its significance test, 2016

\begin{tabular}{|c|c|c|c|c|c|}
\hline \multicolumn{2}{|c|}{ Demographic Characteristics } & Frequency & Percent & \multirow{2}{*}{$\begin{array}{c}\text { Sleep Quality } \\
\text { Mean (SD) }\end{array}$} & \multirow{2}{*}{$\begin{array}{c}\mathbf{P} \\
r=0.044 \\
* P=0.676\end{array}$} \\
\hline Age & Mean (SD) & \multicolumn{2}{|c|}{$55.83 \pm 15.86$} & & \\
\hline \multirow[b]{2}{*}{ Sex } & Male & 51 & 54.80 & $52.54 \pm 15.89$ & \multirow[b]{2}{*}{$* * \mathrm{P}=0.418$} \\
\hline & Female & & 45.20 & $55.52 \pm 18.67$ & \\
\hline \multirow[b]{2}{*}{ Marital status } & Single & 26 & 27.90 & $53.37 \pm 14.91$ & \multirow[b]{2}{*}{$* * \mathrm{P}=0.98$} \\
\hline & Married & 67 & 72.10 & $53.45 \pm 16.59$ & \\
\hline \multirow{3}{*}{$\begin{array}{l}\text { Number of } \\
\text { children }\end{array}$} & One or two & 35 & 37.60 & $56.51 \pm 12.34$ & \multirow{3}{*}{$* * * \mathrm{P}=0.217$} \\
\hline & Three or four & 27 & 29.00 & $55.31 \pm 18.22$ & \\
\hline & $5 \leq$ & 31 & 33.40 & $56.25 \pm 9.54$ & \\
\hline \multirow{4}{*}{$\begin{array}{l}\text { Employment } \\
\text { status }\end{array}$} & Unemployed & 12 & 12.90 & $54.82 \pm 17.61$ & \multirow{4}{*}{$* * * \mathrm{P}=0.794$} \\
\hline & Employed & 27 & 29.00 & $51.01 \pm 17.67$ & \\
\hline & Housewife & 33 & 35.50 & $56.54 \pm 18.15$ & \\
\hline & Retired & 21 & 22.60 & $53.48 \pm 13.69$ & \\
\hline \multirow{5}{*}{ Level of education } & Illiterate & 21 & 22.80 & $53.23 \pm 18.92$ & \multirow{5}{*}{$* * * \mathrm{P}=0.617$} \\
\hline & Reading and writing & 21 & 22.80 & $57.31 \pm 15.88$ & \\
\hline & $\begin{array}{l}\text { Under high school } \\
\text { diploma }\end{array}$ & 17 & 18.50 & $48.85 \pm 18.44$ & \\
\hline & High school diploma & 19 & 20.70 & $56.16 \pm 16.83$ & \\
\hline & University degrees & 15 & 15.20 & $54.12 \pm 14.44$ & \\
\hline \multirow{3}{*}{ Economic status } & Sufficient & 27 & 29.00 & $56.35 \pm 18.24$ & \multirow{3}{*}{$* * * \mathrm{P}=0.479$} \\
\hline & Some what sufficient & 36 & 38.80 & $54.52 \pm 17.22$ & \\
\hline & Insufficient & 30 & 32.20 & $50.86 \pm 15.04$ & \\
\hline \multirow{3}{*}{ Smoking status } & Yes & 11 & 11.80 & $58.20 \pm 19.83$ & \multirow{3}{*}{$* * * \mathrm{P}=0.591$} \\
\hline & No & 70 & 75.30 & $53.89 \pm 17.06$ & \\
\hline & Quitted & 12 & 12.90 & $50.95 \pm 13.32$ & \\
\hline
\end{tabular}

* Pearson Correlation; ** Independent T-Test; *** Kruskal Wallis Test

that the prevalence of sleep disorders in coronary patients admitted to Intensive Care Units was high (81\%) (Sepahvand 2014). Poor sleep quality among patients in care units is associated with frequent abnormalities, changes in circadian rhythm, and reduction in depth and restoration phase of sleep (Beltrami et al., 2015). Patients admitted to the Intensive Care Unit suffered from a severe sleep disorder, an abnormal increase in 1 and 2 sleep stages, reduced night time sleeping, and lack of deep sleep (Hardin 2009).
Moreover, $21 \%$ of patients with myocardial infarction in the Intensive Care Unit have difficulty falling asleep and continuous sleep (Johansson et al., 2010).

Also, based on the results of this study, there was a significant difference between the sleep quality at night before admission and that on the first night of admission; a decrease in the quality of sleep was observed. In addition, there was a positive and significant relationship between the sleep quality on the first night 
Table 2. Numerical indicators of sleep quality in terms of type of disease and its significance test, 2016

\begin{tabular}{ccccc}
\hline Cause of admission & Frequency & Percent & Mean (SD) & P \\
\hline Acute coronary syndrome & 58 & 62.41 & $54.60 \pm 13.64$ & \\
Heart failure & 11 & 11.80 & $53.84 \pm 17.87$ & $\mathrm{P}=0.853$ \\
Acute pulmonary edema & 8 & 8.60 & $52.96 \pm 15.85$ & \\
Others & 16 & 17.20 & $54.43 \pm 17.38$ & Client-Centered Nursing Care
\end{tabular}

Table 3. Frequency distribution of sleep quality of the subjects on the first night of admission, 2016

\begin{tabular}{cl}
\hline Disorder in & Mean (SD) \\
\hline Sleeping depth & $60.35 \pm 29.65$ \\
Ease of falling asleep & $61.79 \pm 29.37$ \\
Waking up frequently & $62.85 \pm 22.21$ \\
Ease of returning to sleep & $63.97 \pm 30.16$ \\
Quality of mental sleep & $64.48 \pm 25.39$ \\
\hline
\end{tabular}

of admission and that on the night before admission. It means that patients with poor quality of sleep before admission experienced poorer sleep quality after admission. It seems that acute conditions of the disease, disruptive environmental factors such as light and sound, and the lack of emotional support from family due to their distance worsened their sleep quality. Patients admitted to the Intensive Care Unit are subject to excessive hearing and visual stimuli (Gholamhoseininejad et al., 2012).

On the one hand, the simultaneous incidence of mental and physical illness increases complaints of sleepless in these patients. On the other hand, being hospitalized and away from the family causes emotional stress in patients, which exacerbates these changes because hospitalized patients spend a long time in bed and in solitude that affects the circadian rhythm and nighttime sleep (Adib-Hajbaghery et al., 2012). Previ- ous studies showed that the sleep quality of patients in hospital is lower than those at home (Ahmadi \& Poormansouri 2015; Bihari et al. 2012; Li et al. 2011; Little et al. 2012; Tamburri et al. 2004). In line with the present study, a study compared the quality of sleep before and after the admission of the patients to the Intensive Care Unit and concluded that the sleep quality became worse after admission to the Intensive Care Unit (Stewart et al., 2017).

Another study showed that the sleep quality at night after admission was worse than before admission, declining from $57 \%$ to $55.3 \%$ (Park et al., 2014). This issue illustrates the importance of addressing the quality of sleep in patients before admission and its effect in the formulation of post-admission care programs. In addition, in the pre-admission study, patients did not have a good sleep quality due to their acute conditions, which became worse after admission. Environmental

Table 4. Sleep quality of the subjects at night before admission and the first night of admission, 2016

\begin{tabular}{cc}
\hline Sleep Quality & Mean (SD) \\
\hline Before admission & $63.68 \pm 17.96$ \\
In the hospital & $54.02 \pm 16.90$ \\
Paired t-test & $\mathrm{t}=6.704, \mathrm{P}<0.0001$ \\
Pearson correlation test & $\mathrm{r}=0.606, \mathrm{P}<0.0001$ \\
\hline
\end{tabular}


stimuli in the Intensive Care Unit have a major role in the sleep disorder of patients (Stewart et al., 2017). Therefore, it is necessary to further study the role of each environmental and individual factor in sleep disorder of patients admitted to the Intensive Care Unit in order to prioritize, as appropriate, the plans to moderate the effective factors.

Limitations of this study were small sample size and low age group of patients under study. It is suggested that further studies should examine the sleep quality of patients over sixty years of age and with larger sample size. In general, the patients in the Intensive Care Unit experienced poor sleep quality. Managers and members of the care team can play an important role in improving the quality and quality of sleep in patients by correctly planning and taking the necessary steps, given their central role in providing patients with comfort. Patients' sleep quality should be evaluated at the time of admission to the Intensive Care Unit and after clearance from the hospital and home accommodation. According to the assessment, a care plan should be developed for them by care unit nurses. To improve the sleep quality of patients in hospital wards, caregivers should take the necessary measures to improve the quality of sleep by controlling or eliminating factors (especially environmental factors such as sound and light) that cause sleep disorders in patients.

\section{Acknowledgments}

This article is extracted from the MSc. thesis of the second Author in the Department of Critical Care Nursing, School of Nursing and Midwifery, Iran University of Medical Sciences of Tehran (IR.IUMS.REC.1394.9311449008). It is also the result of a research project approved by the Iran University of Medical Sciences with the code of ethics IR.IUMS.REC.1394.9311449008 on 2015. We appreciate all the subjects who participated in this research. We are also grateful to the officials and staff of the Faculty of Nursing and Midwifery of the Iran University of Medical Sciences and the research environments that provided the opportunity to conduct the research. We thank all the researchers who collaborated with us for the collection of data.

\section{Conflict of Interest}

The authors declare no conflicts of interest.

\section{References}

Achury-Saldaña, D. M. \& Achury, L. F, 2010. [Sleep in the Critical Patient: An Unmet Need in the Intensive Care Unit (Spanish)]. Nursing Research: Image and Development ,12(1), pp. 25-42.

Adib Hajbaghery, M., Izadi Avanji, F. \& Akbari, H., 2012. Quality of sleep and its related risk factors in hospitalized older patients in Kashan's hospitals, Iran 2009. Iranian Journal of Nursing and Midwifery Research, 17(6), pp. 414-20. [PMID] [PMCID]

Ahmadi, M. \& Poormansouri, S., 2015. Effectiveness of selfmanagement educational program on fatigue in sickle cell patients. Journal of Clinical Nursing and Midwifery, 4, pp. 29-42.

Ahmed, M. A., Youssef, W. \& El Feky, H., 2015. Sleep quality as perceived by critically ill patients at El Manial University Hospitals. Egyptian Journal of Nursing, 10(1). doi: 10.1999/1307$6892 / 34478$

BaHammam, A. et al., 2010. Circadian pattern of sleep, energy expenditure, and body temperature of young healthy men during the intermittent fasting of Ramadan. Appetite, 54(2), pp. 426-429. [DOI:10.1016/j.appet.2010.01.011]

Beltrami, F. G. et al., 2015. Sleep in the intensive care unit. Jor nal Brasileiro de Pneumologia, 41(6), pp. 539-46. [DOI:10.1590/ s1806-37562015000000056

Bihari, S. et al., 2012. Factors affecting sleep quality of patients in intensive care unit. Journal of clinical sleep medicine: JCSM: Official Publication of the American Academy of Sleep Medicine, 8(3), pp. 301-7. [DOI:10.5664/jcsm.1920]

Chandola, T. et al., 2010. The effect of short sleep duration on coronary heart disease risk is greatest among those with sleep disturbance: A prospective study from the Whitehall II cohort. Sleep, 33(6), pp. 739-44. [DOI:10.1093/sleep/33.6.739]

Duong Coburn, N., 2013. A systematic review of sleep quality and sleep promotion in the intensive care unit. San Marcos, California: California State University San Marcos.

Elliott, R., McKinley, S. \& Cistulli, P., 2011. The quality and duration of sleep in the intensive care setting: An integrative review. International Journal of Nursing Studies, 48(3), pp. 384-400. [DOI:10.1016/j.ijnurstu.2010.11.006]

Fontana, C. J. \& Pittiglio, L. I., 2010. Sleep deprivation among critical care patients. Critical Care Nursing Quarterly, 33(1), pp. 75-81. [DOI:10.1097/CNQ.0b013e3181c8e030]

Gami, A. S. et al., 2013. Obstructive sleep apnea and the risk of sudden cardiac death. Journal of the American College of Cardiology, 62(7), pp. 610-6. [DOI:10.1016/j.jacc.2013.04.080]

Gholamhoseininejad, N. et al., 2012. A study of the effective factors on sleep health in hospitalized patients. Journal of Health and Care, 14(1), pp. 44-54

Hardin, K. A., 2009. Sleep in the ICU. Chest, 136(1), pp. 284-94 [DOI:10.1378/chest.08-1546]

Johansson, I. et al., 2010. Disturbed sleep, fatigue, anxiety and depression in myocardial infarction patients. European Journal of Cardiovascular Nursing, 9(3), pp. 175-80. [DOI:10.1016/j.ejce nurse.2009.12.003]

Kamdar, B. B. et al., 2013. The effect of a quality improvement intervention on perceived sleep quality and cognition in a medi- 
cal ICU. Critical Care Medicine, 41(3), pp. 800-9. [DOI:10.1097/ CCM.0b013e3182746442]

Kendzerska, T. et al., 2014. Untreated obstructive sleep apnea and the risk for serious long-term adverse outcomes: A systematic review. Sleep Medicine Reviews, 18(1), pp. 49-59. [DOI:10.1016/j.smrv.2013.01.003]

Kozick, C., 2013. What nurses need to know about sleep. Colorado Nurse, 113(4), p. 4. [PMID]

Li, S. Y. et al., 2011. Efficacy of controlling night-time noise and activities to improve patients' sleep quality in a surgical intensive care unit. Journal of Clinical Nursing, 20(3-4), pp. 396-407. [DOI:10.1111/j.1365-2702.2010.03507.x]

Little, A. et al., 2012. A patient survey of sleep quality in the intensive care unit. Minerva Anestesiologica, 78(4), pp. 406-14. [PMID]

Matthews, E. E., 2011. Sleep disturbances and fatigue in critically ill patients. AACN Advanced Critical Care, 22(3), pp. 204-24. [DOI:10.1097/NCI.0b013e31822052cb]

Monteiro, N. T. \& Ceolim, M. F., 2014. Quality of sleep of the aged at home and in hospital. Texto \& Contexto - Enfermagem, 23(2), pp. 356-64. [DOI:10.1590/0104-07072014000190013]

Neyse, F. et al., 2011. The effect of earplugs on sleep quality in patients with acute coronary syndrome. Journal of Critical Care Nursing, 4(3), pp. 127-34.

Paddock, C., 2013. Sleep may play important role in heart disease [Internet]. viewed 2013 November 20, https:/ / www.medicalnewstoday.com/articles/262812.php

Park, M. J. et al., 2014. Noise in hospital rooms and sleep disturbance in hospitalized medical patients. Environmental Health and Toxicology, 29, p. e2014006. [DOI:10.5620/eht.2014.29. e2014006]

Schiza, S. E. \& Mermigkis, C., 2010. Sleep in acute coronary syndrome patients. Sleep Medicine, 11(8), p. 805. [DOI:10.1016/j. sleep.2010.04.001]

Sepahvand, E., Jalali, R., Khaledi Paveh, B. \& Rezaei, M. 2014 Development and validation of sleep disturbance questionnaire in patients with acute coronary syndrome. International scholarly research notices, 2014. 2014, p. 978580. [DOI:10.1155/2014/978580]

Stewart, J. A. et al., 2017. Factors influencing quality of sleep among non-mechanically ventilated patients in the intensive care unit. Australian Critical Care, 30(2), pp. 85-90. [DOI:10.1016/j.aucc.2016.02.002]

Tamburri, L. M. et al., 2004. Nocturnal care interactions with patients in critical care units. American Journal of Critical Care, 13(2), pp. 102-13. [PMID]

Tobaldini, E. et al., 2017. Sleep, sleep deprivation, autonomic nervous system and cardiovascular diseases. Neuroscience $\mathcal{E}$ Biobehavioral Reviews, 74, pp. 321-9. [DOI:10.1016/j.neubii orev.2016.07.004]

Zeighami, S. M. \& Shahparian, M., 2013. [Evaluation of sleep problems and its associated factors in male patients with systolic heart failure (Persian)]. Qom University of Medical Sciences Journal, 6(4), pp. 64-73. 
August 2017 . Volume 3. Number 3

Client-Centered Nursing Care 